\title{
COMMENTARY
}

\section{Controversial Messages on Cancer}

\section{Vicente Mustieles ${ }^{1}$, Nicolas Olea $^{1,2}$, Maria Jose Sanchez ${ }^{2,3}$, Mariana F Fernandez $^{1,2 *}$}

\begin{abstract}
A direct connection between certain lifestyle factors and an increased risk of cancer has already been established. Despite well-documented associations, controversial messages on causes of cancer often appear without taking into consideration their impact on the general population. While mainstream coverage of cancer research is clearly welcome, consideration must be given to the risk of transmitting provocative messages with serious negative consequences for cancer research and public health. We must avoid becoming a breeding ground for the misinterpretation of scientific information. Polemical results can stimulate scientific research and progress, but controversial messages encourage confusion and impotence in an increasingly disoriented population. The correct communication of health messages is at least as important as research on risk factors.
\end{abstract}

Keywords: Cancer - prevention - lifestyle - communication of health messages

Asian Pac J Cancer Prev, 16 (14), 6171-6172

\section{Introduction}

A direct connection has been established between an unhealthy diet or lifestyle and an increased risk of cancer and other chronic diseases (Wiseman, 2008). The overall burden of cancer morbidity and mortality remains substantially dependent on behavioral and environmental risk factors, including tobacco smoking, unhealthy diets, and physical inactivity, as well as cancer-related infectious diseases (Stewart and Wild, 2014). Around one-third of cancers in high-income Western societies have been attributed to factors related to food, nutrition, and physical activity (WCRF/AICR, 2007; Romaguera et al., 2012). Furthermore, developing countries are showing the same trend for several types of cancers, partially due to adoption of western lifestyles (Park et al., 2008).

Because more than half of all cancer cases can be prevented (IARC, 2015), international agencies have focused on minor lifestyle changes to reduce the risk (World Cancer Research Fund International; European Code Against Cancer), including the avoidance of tobacco, the moderation of alcohol intake, and the achievement of a good nutritional status and healthy weight (based on a balanced diet and regular physical activity) through a diet rich in plant-based foods and poor in red/processed meat and salty foods (Romaguera et al., 2012).

Despite these well-documented associations, it was recently suggested that the main contributor to cancer overall is the "bad luck" associated with random mutations rather than poor lifestyle choices (Tomasetti and Vogelstein, 2015). The article engendered headlines in the communications and social media on "The bad luck of cancer"; "Most cancer types 'just bad luck", among many others, reflecting a very superficial reading of the publication, facilitated by an abstract that failed to acknowledge the serious weaknesses of the study (Tomasetti and Vogelstein, 2015). The highly frequent coverage given to this type of controversial message on cancer causes appears to take no consideration of their impact on the lifestyle and habits of the general population. For instance, a recent epidemiological study made the astonishing claim that the vegetarian diet is associated with worse health outcomes (higher incidence of cancer, allergies, and mental health disorders), a greater need for health care, and a poorer quality of life (Burkert et al., 2014). The validity of such a conclusion depends on multiple factors, including the statistical power of the study, its freedom from bias, and the proper weighting of accumulated evidence from other investigations on the same research issue (Ioannidis, 2005). The aforementioned studies suffer from various shortcomings, some of which are acknowledged by the authors.

One key question is whether the interpretations given in the abstracts truly reflect the limitations of these studies (Burkert et al., 2014; Tomasetti and Vogelstein, 2015). The weaknesses of the study on vegetarian diets include: its cross-sectional design, preventing the drawing of causal inferences; the self-reporting of dietary habits and lack of data on previous diets; the grouping together of vegans, lacto-ovo-vegetarians and pesco-vegetarians in a "vegetarian group" due to sample size limitations, with the majority of this group regularly consuming fish; and the self-reporting of their health status using questionnaires, with no clinical verification (Burkert et al., 2014). Because the latency period of chronic diseases can be long, lifetime dietary habits may be more relevant than the current or recent diet. Crucially, the adoption of a given dietary pattern may be a response to a disease rather

${ }^{1}$ University of Granada, ${ }^{2}$ CIBER de Epidemiología y Salud Pública (CIBERESP), ${ }^{3}$ Andalusian School of Public Health, Granada Cancer Registry, Instituto de Investigación Biosanitaria ibs.Granada, Spain*For correspondence: marieta@ugr.es 
than its cause. In other words, could the onset of cancer among study participants prompt a modification in dietary habits from non-vegetarian to vegetarian? In this line, another study found that $75 \%$ of a group of vegetarians with cancer had changed to this diet after the diagnosis (Gilsing et al., 2013).

While mainstream coverage of cancer research is evidently welcome, consideration must be given to the risk of transmitting a sensationalist message with serious negative consequences for cancer research and public health. Faced by contradictory information, individuals must rely on their instincts for evaluating potential threats (Brown, 2014; Tsuchiya 2015). Colon it is especially important to be frank about the limits of our knowledge and to tell people when no answer is available. For example, the identification of specific relationships between diet and cancer is highly challenging, due to the lengthy latency and complex pathogenesis of many cancers. However, if there is something we have learned about this disease, it is that prevention is the most costeffective strategy for its control and must be addressed from all possible perspectives (Vineis, 2014).

Given the wide and increasing public interest in the relationship between cancer and dietary habits, it appears essential to alert readers to the limitations of studies of this type and warn them of the need to interpret the data with due caution. It is of particular importance to ensure that the abstracts of papers honestly reflect their findings and weaknesses. We highlight the need for well-designed longitudinal studies on the relationship between an unhealthy diet or lifestyle and health in order to offer solid evidence to healthcare professionals and the public. The success of international efforts to prevent chronic diseases such as cancer depends on the coherence and proper communication of high-quality messages to promote healthy lifestyles in the general population. Above all, we must avoid becoming a breeding ground for the misinterpretation of scientific information. Polemical results can stimulate scientific research and progress, but controversial messages encourage confusion and impotence in an increasingly disoriented general population. The correct communication of health messages is at least as important as research on risk factors. If we fail in health messages to the population, we fail in everything (Figure).

Future investigation of risk factors will determine the effectiveness and impact of cancer prevention campaigns, the most effective weapon in the long-term fight against this evil of modern societies. The World Cancer Day 2015

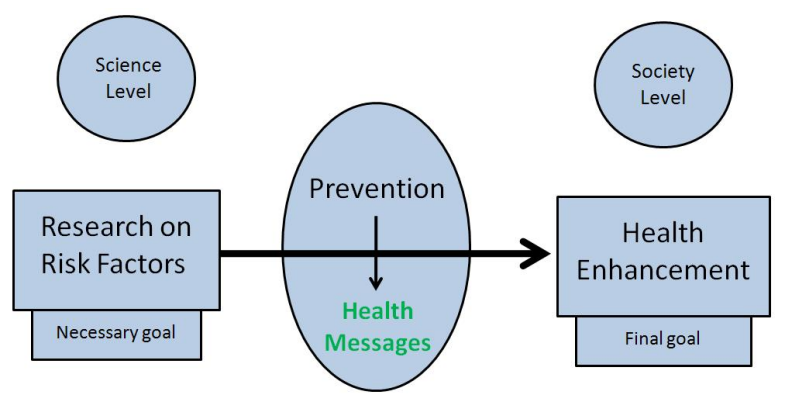

Figure. If We Fail in Health Messages to the Population, We Fail in Everything message proposed by the World Cancer Research Fund is "Cancer Prevention: together we can", as an initiative to engage a global audience with its core message that cancer is preventable.

\section{Acknowledgements}

The authors are grateful to Richard Davies for editorial assistance. This study was supported by the Spanish Ministry of Health (CIBERESP and FIS PI11/0610 and PI13/02406) and the Andalusia Regional Government, Council of Innovation, Science and Enterprise (Excellence Project P09-CTS-5488) and Council of Health (SAS PI0675-2010).

\section{References}

Brown VJ (2014). Risk perception: it's personal. Environ Health Perspect, 122, 276-9.

Burkert NT, Muckenhuber J, Großschädl F et al. (2014). Nutrition and health - the association between eating behavior and various health parameters: a matched sample study. PLoS One, 9, 88278.

European Code Against Cancer. 12 ways to reduce your cancer risk. http://cancer-code- europe.iarc.fr/images/doc/ ecac_en.pdf

Gilsing AM, Weijenberg MP, Goldbohm RA et al. (2013). The Netherlands Cohort Study-Meat Investigation Cohort; a population-based cohort over-represented with vegetarians, pescetarians and low meat consumers. Nutr J, 12, 156.

IARC (2015). Most types of cancer not due to "bad luck". IARC responds to scientific article claiming that environmental and lifestyle factors account for less than one third of cancers. http://www.iarc.fr/en/media-centre/pr/2015/pdfs/ pr231_E.pdf

Ioannidis JP (2005). Why most published research findings are false. PLoS Med, $2,124$.

Park S, Bae J, Nam BH, Yoo KY (2008). Aetiology of cancer in Asia. Asian Pac J Cancer Pre, 9, 371-80.

Romaguera D, Vergnaud AC, Peeters PH et al. (2012). Is concordance with World Cancer Research Fund/American Institute for Cancer Research guidelines for cancer prevention related to subsequent risk of cancer? Results from the EPIC study. Am J Clin Nutr, 96, 150-63.

Stewart BW, Wild CP, editors (2014). World Cancer Report 2014 Lyon, France: International Agency for Research on Cancer.

Tomasetti C, Vogelstein B (2015). Cancer etiology. Variation in cancer risk among tissues can be explained by the number of stem cell divisions. Science, 347, 78-81.

Tsuchiya M (2015). Lay beliefs, knowledge, and attitudes towards cancer: a pilot study in Japan. Asian Pac J Cancer Prev, 16, 3247-51.

Vineis P, Wild CP (2014). Global cancer patterns: causes and prevention. Lancet, 383, 549-57.

Wiseman M (2008). The second World Cancer Research Fund/ American Institute for Cancer Research expert report. Food, nutrition, physical activity, and the prevention of cancer: a global perspective. Proc Nutr Soc, 67, 253-6.

World Cancer Research Fund / American Institute for Cancer Research. Food, Nutrition, Physical Activity, and the Prevention of Cancer: a Global Perspective (2007). http:// www.dietandcancerreport.org/cancer_resource_center/ downloads/Second_Expert_Report_full.pdf

World Cancer Research Fund International. Cancer Prevention Recommendations. http://www.wcrf.org/int/research-wefund/our-cancer-prevention-recommendations 